\title{
INFLUENCIA DE LA NAO EN LA COVARIABILIDAD DE TEMPERATURAS Y PRECIPITACIONES DE INVIERNO EN ESPAÑA, 1946-2005
}

\author{
Fernando S. RODRIGO ${ }^{1}$ \\ ${ }^{1}$ Dpto. Química y Física, Universidad de Almería \\ frodrigo@ual.es
}

\section{RESUMEN}

En este trabajo se analiza la influencia de la Oscilación del Atlántico Norte (NAO) en la covariabilidad de temperaturas y precipitaciones de invierno de 18 estaciones meteorológicas distribuidas por el territorio peninsular español durante el periodo 1946-2005. La covariabilidad entre precipitaciones totales acumuladas (R) y media estacional de las temperaturas máximas diarias (TX), mínimas diarias (TN), medias diarias (TM), y del rango diurno de temperaturas (DTR), fue analizada comparando los resultados obtenidos para las distintas fases de la NAO. Entre las variables relacionadas con la temperatura, la que muestra mayor cobertura espacial de resultados estadísticamente significativos es la DTR. Entre los principales resultados se obtiene el descenso (aumento) de los valores medios de DTR durante la fase NAO negativa (positiva), aunque no hay cambios importantes en las correlaciones entre variables dependiendo de las fases de la NAO. En términos generales, y durante la fase NAO negativa (positiva), se produce un aumento (descenso) de la covariabilidad de temperaturas y precipitaciones en las estaciones occidentales, mientras que el comportamiento opuesto se detecta en las estaciones del litoral mediterráneo.

Palabras clave: NAO, temperaturas, precipitaciones, covariabilidad

\begin{abstract}
The influence of the North Atlantic Oscillation (NAO) on the covariability of temperatures and precipitation in 18 stations of the Iberian Peninsula during the period 1946-2005 is analyzed in this work. Daily data of minimum, maximum, mean temperature, and daily temperature range were used to obtain seasonal means (TN, TX, TM, and DTR, respectively), and the covariability of these variables with accumulated seasonal rainfall $(\mathrm{R})$ was studied comparing results obtained for different NAO phases. The variable that shows the greatest spatial coverage of statistically significant results is the DTR. It has been detected a decrease (increase) of DTR during the negative (positive) NAO phase, although there is not important differences of the correlations depending on the NAO phases. In general terms, during the negative (positive) NAO phase, there is an increase (decrease) of the covariability of temperatures and rainfall in western stations, while the opposite behavior is found in the Mediterranean stations.
\end{abstract}

Key words: NAO, temperatures, rainfall, covariability. 


\section{INTRODUCCIÓN}

Es bien conocida la relación entre la Oscilación del Atlántico Norte (NAO) y las precipitaciones en la Península Ibérica (Goodess and Jones, 2002). Son pocos, sin embargo, los estudios que analizan posibles relaciones entre la NAO y las temperaturas en el Sur de Europa (Pozo-Vázquez et al., 2001; Castro-Díez et al., 2002; Trigo et al., 2002), y, que sepamos, no hay análisis en la literatura centrados en la covariabilidad de temperaturas y precipitaciones bajo distintas fases de la NAO.

En invierno, y durante la fase positiva de la NAO, las condiciones anticiclónicas predominantes están asociadas con una reducida nubosidad, y por tanto mayor radiación solar durante el día, y mayor pérdida de radiación de onda larga durante la noche (al contrario durante la fase NAO negativa). Esto llevaría a un aumento (disminución) de las temperaturas máximas (mínimas) durante la fase NAO positiva (lo contrario durante la NAO negativa). El resultado sería una correlación negativa (positiva) entre temperaturas máximas (mínimas) y precipitaciones. No obstante, la covariabilidad entre temperaturas y precipitaciones también responde a otros mecanismos físicos relacionados con la humedad del suelo y los flujos de calor sensible y calor latente, así como las fluctuaciones de la circulación atmosférica (Trenberth and Shea, 2005).

El objetivo de este trabajo es analizar la covariabilidad de precipitaciones y temperaturas bajo las distintas fases de la NAO en un conjunto de 18 estaciones meteorológicas distribuidas por la Península Ibérica.

\section{DATOS Y MÉTODOS}

La base de datos usada en este estudio comprende cantidades mensuales de precipitación y temperaturas diarias mínimas, máximas y medias para 18 localidades españolas que cubren los principales dominios climáticos de la Península Ibérica durante el periodo 1946-2005. La Figura 1 muestra la localización de las estaciones. Los datos mensuales de lluvia fueron extraídos de la base de datos homogeneizada por la Agencia Estatal de Meteorología (AEMET, Luna et al., 2002) y los datos de temperatura de la base de datos Spanish Daily Adjusted Temperature Series (SDATS, Brunet et al., 2006). Estas bases de datos están disponibles bajo el epígrafe "Series largas de referencia" en la página web de la AEMET (http://www.aemet.es). El rango diario de temperaturas fue calculado a partir de los datos diarios, y luego se obtuvieron los valores medios estacionales de las variables de temperatura: TN (media estacional de las mínimas diarias), TX (máximas), TM (medias), DTR (rango diurno de temperaturas). Los datos mensuales de lluvia fueron usados para obtener el total de precipitaciones $(\mathrm{R})$ de invierno, considerado éste como es usual, de Diciembre a Febrero. Los datos del índice NAO corresponden al índice de Hurrell (Hurrell et al., 2003) y están disponibles en la página web del National Center for Atmospheric Research (NCAR, htpps://climatedataguide.ucar.edu/climate-data/). La Figura 2 muestra la serie temporal de este índice para el periodo de estudio.

El conjunto de estaciones utilizado es representativo de los diferentes dominios climáticos existentes en la Península Ibérica (Martín Vide y Olcina Cantos, 2001), incluyendo estaciones situadas en la costa norte, la vertiente mediterránea, la Meseta central y el sur de la Península. Para cada estación se calcularon las anomalías estandarizadas de cada variable usando como periodo de referencia el periodo 1961-1990. 
Para distinguir las distintas fases de la NAO se determinó el valor de la mediana (-0.2), y se designó como NAO negativa (NAO-) a aquel valor $<-0.2$, y como NAO positiva $(\mathrm{NAO}+)$ a aquel valor $\geq-0.2$. Aunque esta división engloba en una misma categoría condiciones "normales" y extremas de la NAO, permite contar con muestras suficientemente grandes de datos (32 años con $\mathrm{NAO}+, 28$ con NAO-), de cara a los estudios estadísticos.

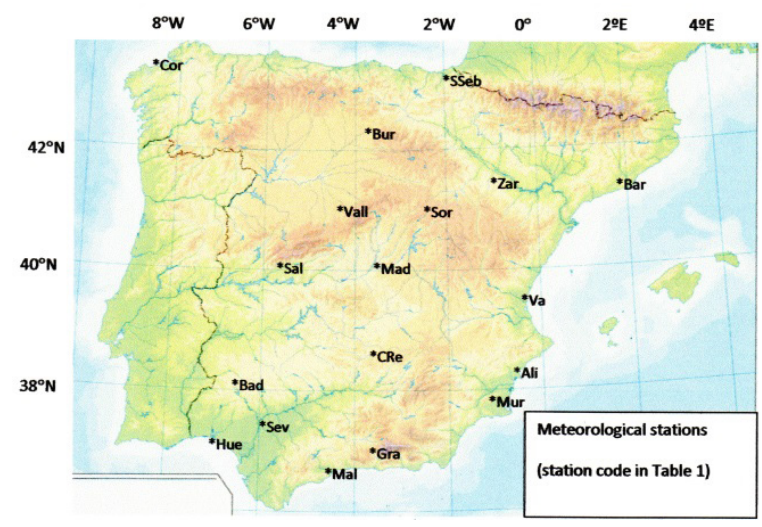

Fig. 1. Estaciones meteorológicas utilizadas en este estudio.

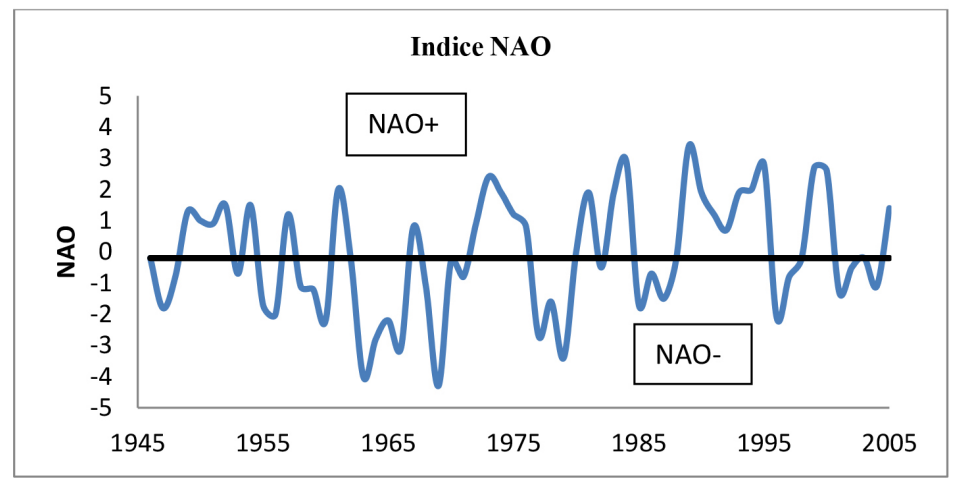

Figura 2. Indice NAO de Hurrell, 1946-2005. La linea horizontal indica el valor de la mediana (-0.2), que permite distinguir los valores correspondientes a las fases positiva $(\mathrm{NAO+})$ y negativa (NAO-).

Los datos del periodo completo fueron divididos en dos subconjuntos, uno correspondiente a la fase $\mathrm{NAO}+$, y otro a la fase NAO-. A continuación se compararon los valores medios de cada variable usando el test $t$ de diferencia entre las medias. Los coeficientes de correlación entre TN, TX, TM, DTR y R fueron calculados para cada subconjunto. Para el análisis de los cambios en la covariabilidad se utilizó el método de las elipses gaussianas (Rodrigo, 2015). El test de Barnett, que usa como hipótesis nula la igualdad entre las matrices de covarianza de las dos muestras que se comparan, 
fue utilizado para indicar la significación de estas diferencias. Todos los tests fueron estimados al $95 \%$ de nivel de confianza.

\section{RESULTADOS}

La Figura 3 muestra los resultados de aplicar el test $t$ de diferencia entre las medias expresados como la diferencia (NAO-)-(NAO+). Los resultados para $\mathrm{R}$ indican un aumento significativo de las precipitaciones durante la fase NAO- fundamentalmente en las estaciones occidentales, sin cambios significativos en las estaciones mediterráneas y al norte (San Sebastián). Este resultado es conocido y ha sido reflejado en multitud de estudios. En cuanto a las temperaturas, se obtiene un aumento significativo de las temperaturas mínimas bajo la fase negativa, sobre todo en la mitad sur de la Península Ibérica. El menor enfriamiento radiativo como consecuencia de la mayor nubosidad durante la fase NAO- puede explicar este resultado. De igual forma, las tendencias negativas que aparecen en el caso de TX responderían a la relación entre cobertura nubosa y temperaturas máximas. Sin embargo, en este caso, las diferencias sólo son significativas en unas pocas estaciones, y sin un patrón espacial claro. Como resultado, el comportamiento de TM tampoco ofrece resultados estadísticamente significativos, lo cual explica la escasa atención que ha obtenido en la literatura el estudio de la influencia de la NAO en las temperaturas, ya que tradicionalmente se han estudiado las temperaturas medias. Por su parte, el DTR, al sumar el comportamiento de ambas variables, permite obtener una diferencia estadísticamente significativa en la mayoría de las estaciones, con una tendencia a disminuir bajo condiciones de NAO-. Este resultado coincide con los estudios que han detectado en muchas partes del mundo una disminución de la DTR en periodos húmedos (Dai et al., 1999). Bajo condiciones de $\mathrm{NAO}+$, menor nubosidad y menores precipitaciones provocan un aumento de TX y una disminución de TN, con el consiguiente aumento de DTR. Por el contrario, bajo NAO-, con más precipitaciones y nubosidad, se producirá una disminución de TX, un aumento de TN, y, en consecuencia, una disminución de DTR. El balance radiativo en términos de la mayor o menor nubosidad sirve para explicar este comportamiento (Sánchez-Lorenzo et al., 2008). Por otra parte, vemos cómo la variable relacionada con la temperatura que mejor recoge la posible influencia de la NAO es el DTR, con un comportamiento espacialmente homogéneo, sin diferencias regionales.

La Figura 4 muestra los coeficientes de correlación entre $\mathrm{R}$ y cada una de las variables de temperatura, para cada uno de los subconjuntos (NAO+ y NAO-). En primer lugar se observa que apenas existe relación entre TX y R, para ambas fases de la NAO. En invierno, las temperaturas máximas son lo suficientemente bajas como para que la saturación se alcance relativamente pronto, y la relación entre insolación, TX y lluvia sea muy débil, independientemente de la fase de la NAO. Trigo et al. (2002) ya encontraron que la señal NAO es más débil en TX que en TN, un resultado similar al detectado aquí, donde puede apreciarse la correlación positiva entre $\mathrm{R}$ y TN, en ambas fases de la NAO, y fundamentalmente en las estaciones occidentales. Las estaciones de la vertiente mediterránea y la costa norte no presentan correlaciones significativas, reflejando así el conocido patrón espacial de la influencia de la NAO en la Península Ibérica. El comportamiento de TN es el que rige fundamentalmente los resultados de TM, con correlaciones positivas en las estaciones occidentales. En cuanto al DTR, el número de estaciones con correlaciones negativas significativas es mayor, aunque 
nuevamente las estaciones mediterráneas carecen de relaciones significativas. No se aprecian cambios importantes en el orden de magnitud de los coeficientes en función de la fase de la NAO (el test de diferencias entre coeficientes de correlación no proporcionó diferencias estadísticamente significativas, excepto en los casos de Barcelona y Alicante, donde los coeficientes no fueron significativos).
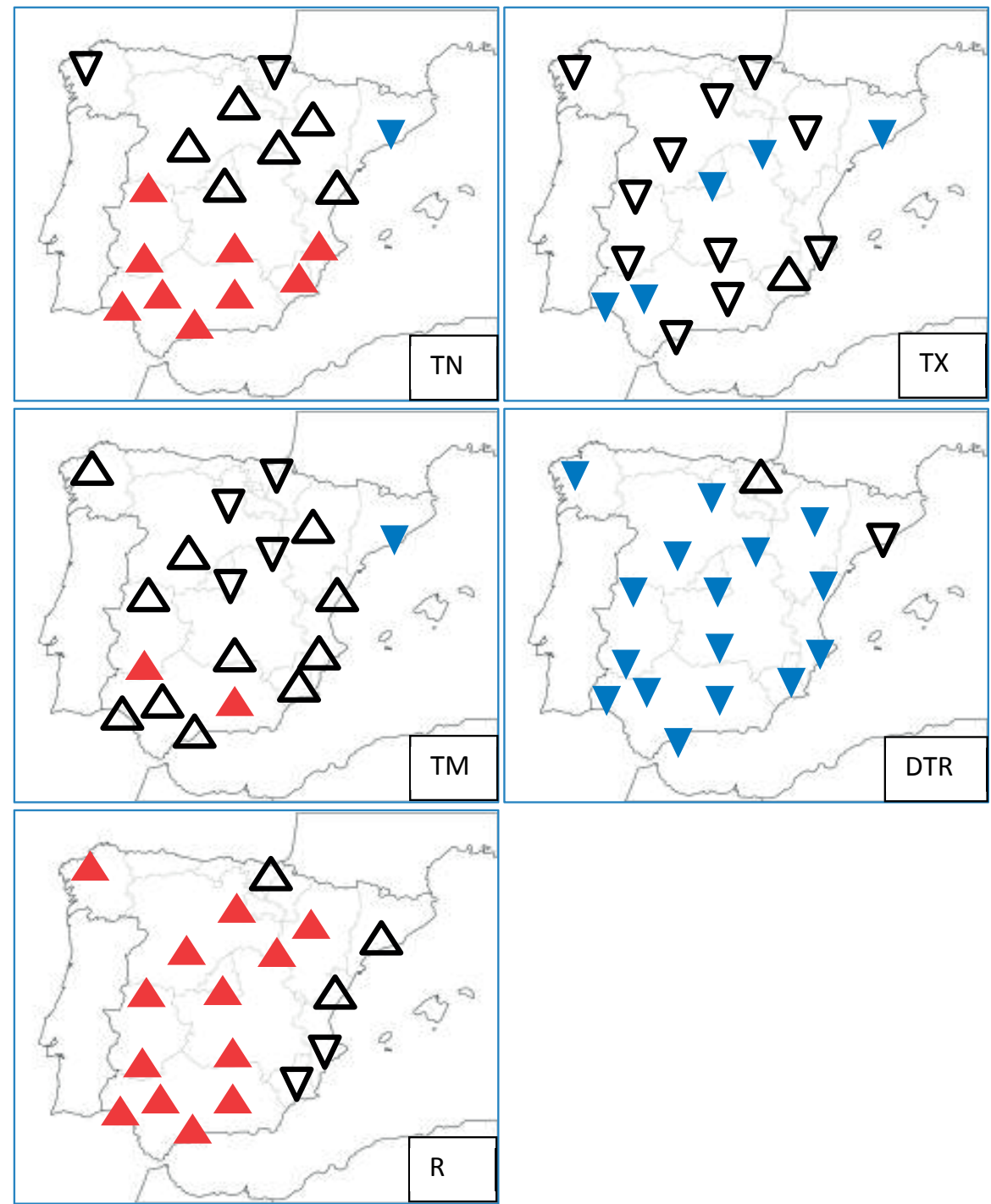

Figura 3. Diferencia de valores medios de las variables de invierno (NAO-)-(NAO+). Triángulos hacia arriba (abajo): diferencia positiva (negativa). En color (sin color) diferencias estadísticamente significativas (no significativas) al nivel de confianza del 95\% según el test $t$ de diferencia entre las medias. 

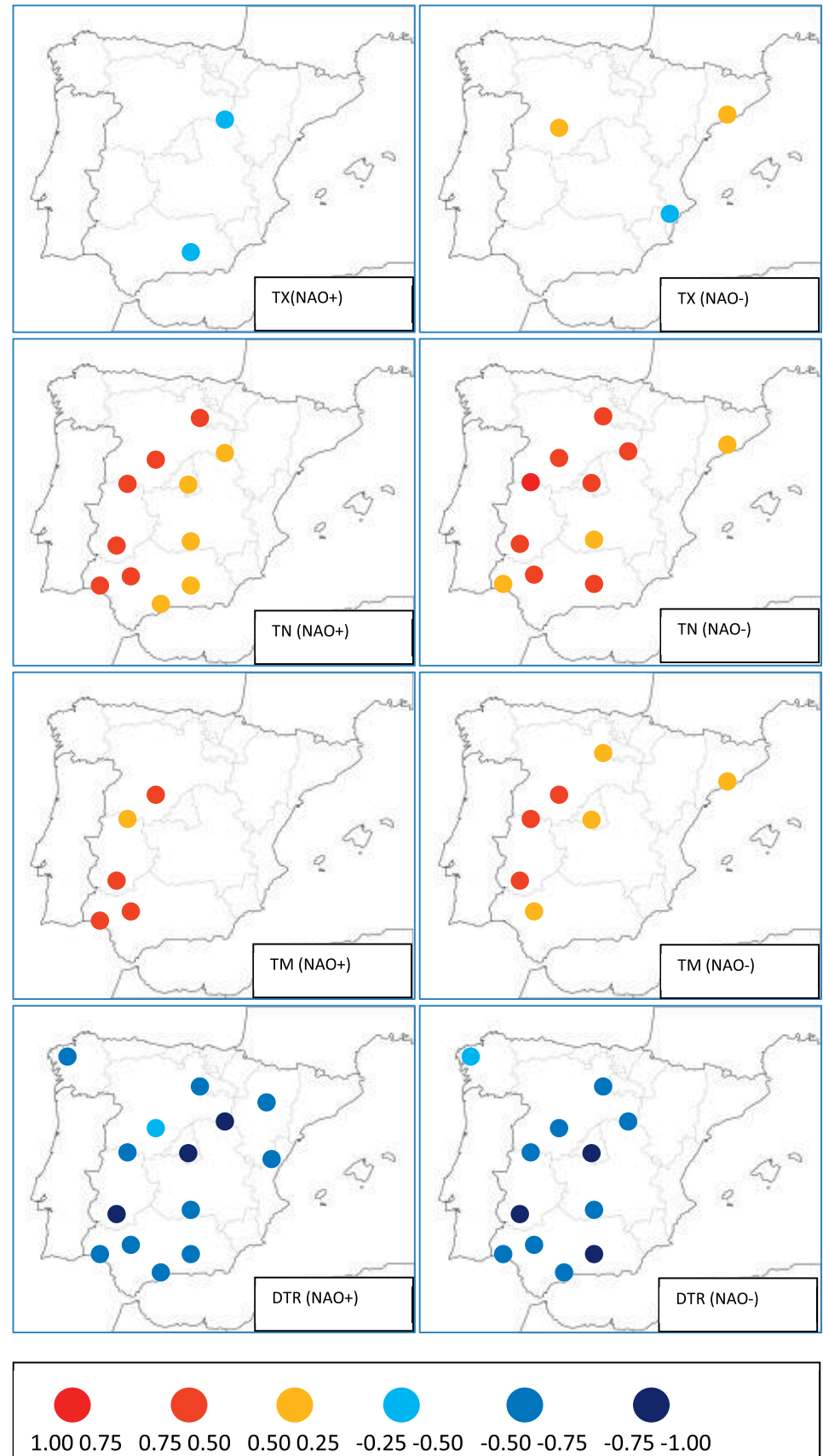

Figura 4. Coeficientes de correlación entre las variables de temperatura y $R$ para las dos fases de la NAO. Sólo se muestran los coeficientes estadísticamente significativos al nivel de confianza del 95\%. 
La Figura 5 muestra los cambios en la covariabilidad entre las variables de temperatura y $\mathrm{R}$, de nuevo expresados como la diferencia (NAO-)-(NAO+).
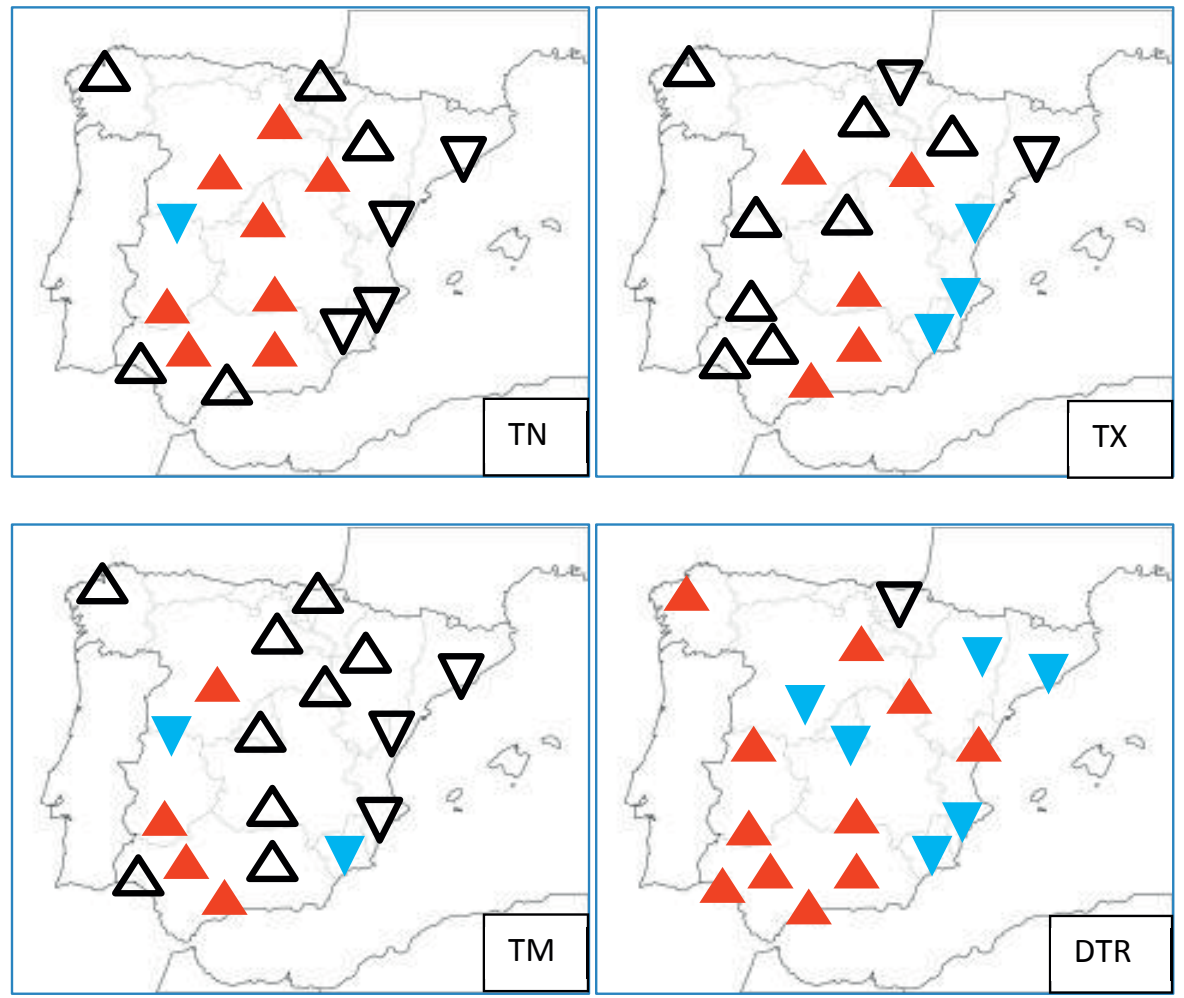

Figura 5. Cambios de la covariabilidad entre las variables de temperatura y $R$, (NAO-)$(\mathrm{NAO}+$ ). Triángulos hacia arriba (abajo): diferencias positivas (negativas). En color (sin color): diferencias estadísticamente significativas (no significativas) al nivel de confianza del 95\%, según el test de Barlett de comparación de las matrices de covarianza.

En términos generales se aprecia un aumento de la covariabilidad en la fase negativa en las estaciones occidentales, tanto para TN como TX, mientras que disminuye de forma significativa en las estaciones mediterráneas para TX. Ambos efectos se compensan parcialmente al analizar el comportamiento de TM (dado que las relaciones entre R y TN y TX son de signo opuesto), mientras que se suman al considerar el comportamiento de DTR. Los cambios en la covariabilidad parecen reflejar el comportamiento de las precipitaciones, en el sentido de que son crecientes allí donde lo son las precipitaciones (Figura 3), fundamentalmente en el área suroccidental.

\section{DISCUSIÓN}

La Figura 6 muestra las elipses de confianza al 95\% correspondientes a ambas fases de la NAO para el DTR en Badajoz, una estación representativa del conjunto afectado por las fases de la NAO, en el sector suroccidental de la Península. 


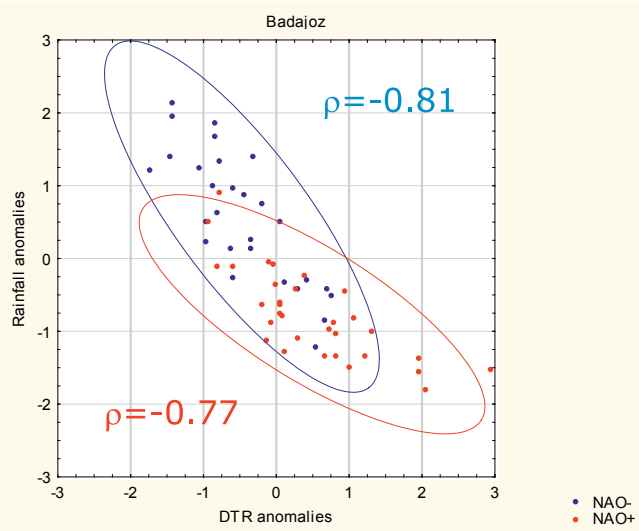

Figura 6. Elipses de confianza al 95\% para R y DTR en Badajoz y ambas fases de la NAO. Se incluyen los coeficientes de correlación $\rho$ en ambos casos.

Puede apreciarse, además de la correlación negativa, cómo los valores medios se desplazan hacia valores positivos de $\mathrm{R}$ y negativos de DTR en la fase NAO-. El vector de valores medios pasa de ser $(-0.47,+0.57)$ para NAO- a $(+0.49,-0.77)$ para $\mathrm{NAO}+$, es decir, en la fase NAO- con el aumento de las precipitaciones se produce un descenso del DTR, como se discutió anteriormente. Según el t-test de diferencia entre las medias, los cambios son significativos al 95\% de nivel de confianza para ambas variables. El cambio del área cubierta por las elipses nos informa de los cambios en la dispersión de los datos alrededor de los ejes principales de la elipse (Rodrigo, 2015). En este caso el cambio es un aumento significativo del 4\% durante la fase NAO-.

A efectos de comparación, las Figura 7 muestra las elipses correspondientes a Alicante, en la costa mediterránea, donde el impacto de la NAO es mucho menor, para las mismas variables, R y DTR.

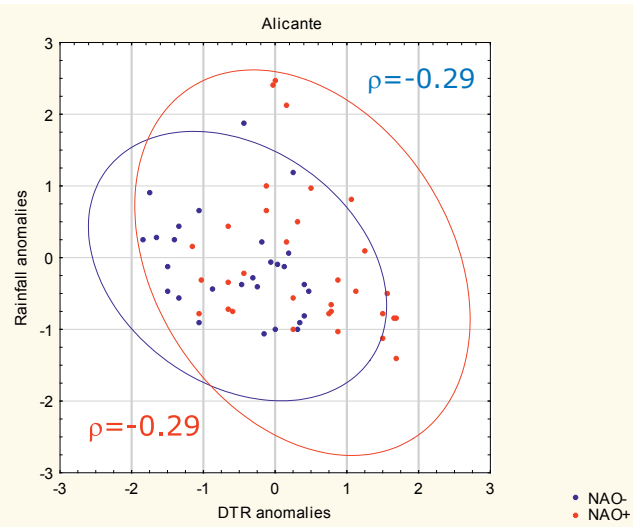

Figura 7. Elipses de confianza al 95\% para R y DTR en Alicante y ambas fases de la NAO. Se incluyen los coeficientes de correlación $\rho$ en ambos casos 
En este caso las correlaciones, aunque ambas negativas, no son significativas. Se observa cómo la diferencia entre los valores medios es mucho menor, pasando de $(+0.38,-0.07)$ durante la fase $\mathrm{NAO}+$ a $(-0.52,-0.12)$ durante la fase NAO-, con diferencias significativas en el caso del DTR y no en el de R. Además, hay un aumento significativo del $44 \%$ en la covariabilidad de la fase NAO+.

\section{CONCLUSIONES}

La influencia de la NAO de invierno en la Península Ibérica no sólo se detecta en el comportamiento de las precipitaciones, sino también en el de las temperaturas, con mayor incidencia en las temperaturas mínimas que en las máximas, y un comportamiento más extendido (incluso hacia las estaciones mediterráneas), de disminución (aumento) del rango diurno de temperaturas durante la fase negativa (positiva) de la NAO. Sin embargo, como se deduce de la comparación de los coeficientes de correlación de ambas fases, no parece que las distintas fases de la NAO afecten a los mecanismos físicos responsables de las relaciones entre precipitaciones y temperaturas. Se detecta un aumento de la variabilidad conjunta de precipitaciones y rango diurno de temperaturas en las estaciones suroccidentales durante la fase negativa de la NAO, mientras se aprecia el comportamiento opuesto en el caso de las estaciones mediterráneas.

\section{REFERENCIAS}

Brunet M, Saladié O, Jones P, Sigró J, Aguilar E, Moberg A, Lister D, Walther A, Lopez D, Almarza C. (2006). The development of a new dataset of Spanish daily adjusted temperature series (SDATS) (1850-2003). Int. J. Climatol., 26, 1777-1802.

Castro-Díez Y, Pozo-Vázquez D, Rodrigo FS, Estreban-Parra MJ. (2002) NAO and winter temperature variability in southern Europe. Geophys. Res. Lett., 29, doi: 10.129/2001GL014042.

Dai A., Trenberth E, Karl TR (1999). Effects of clouds, soil moistrure, precipitation, and wáter vapor on diurnal temperature range. J. Climate., 12, 2451-2473.

Goodess C, Jones PD. (2002) Links between circulation and changes in the characteristics of Iberian rainfall. Int. J. Climatol., 22, 1593-1615.

Hurrell JW, Kushnir Y, Ottersen G, Visbeck M (Eds). (2003). The North Atlantic Oscillation: Climate significance and environmental impact. Washington: American Geophysical Union.

Luna MY, Guijarro JA, López JA. (2012). A monthly precipitation database for Spain (1851-2008): Reconstruction, homogeneity and trends. Advances in Science and Research, 8, 14. Doi:10.5194/asr-8-1-2012

Martín Vide J, Olcina Cantos J. (2001). Climas y tiempos de España. Madrid: Alianza Editorial.

Pozo-Vázquez D, Esteban-Parra MJ, Rodrigo FS, Castro-Díez Y. (2001). A study of NAO variability and its posible non-linear influences on European surface temperatures. Clim. Dyn., 17, 701-715

Rodrigo FS. (2015). On the covariability of seasonal temperatures and precipitation in Spain, 1956-2005. Int. J. Climatol., 35, 3362-3370. 
Sánchez-Lorenzo A, Sigró J, Calbó J, Martín-Vide J, Brunet M, Aguilar E, Brunetti M. (2008). Efectos de la nubosidad e insolación en las temperaturas recientes de España. In: Sigró Rodríguez J, Bruner India M, Aguilar Frons E (Eds) Cambio Climático Regional y sus Impactos, pp. 273-284, Tarragona: Asociación Española de Climatología.

Trenberth KE, Shea DJ. (2005) Relationships between precipitation and surface temperature. Geophys. Res. Lett., 32, L14703. Doi: 10.1029/2005GL022760, 2005.

Trigo RM, Osborn TJ, Corte-Real JM. (2002) The North Atlantic Oscillation influence on Europe: climate impacts and associated physical mechanisms. Clim. Res., 20, 9-17. 